\title{
Compact Printed Log Periodic Dipole Antenna with Second Order Semi-Circle Iteration
}

\author{
Mehmet C. Ozgonul, Mustafa Secmen, Member, IEEE, and Serdar Okuyucu
}

\begin{abstract}
In this study, a size-reduced Log Periodic Dipole Antenna (LPDA) with second order semi-circle iteration is presented. Second order semi-circles are added to the dipole arms, which results in lateral size reduction of $23 \%$ and $8 \%$ with respect to standard LPDA and LPDA with first order semi-circle iteration, respectively. Measurement and simulation results demonstrate that the antenna has a wide range of operating frequency from $0.87 \mathrm{GHz}$ to 2.55 GHz, and shows better gain variation than reference LPDAs.

Keywords - Log periodic antenna, wide-band, printed antenna, size-reduced.
\end{abstract}

\section{INTRODUCTION}

L og Periodic Dipole Antenna (LPDA) is a frequency independent antenna appropriate for use in wideband applications [1]. LPDAs have both acceptable gain and a wide range operating frequency and can be used in many broadband applications to cover the range of frequencies required by the particular application in use. LPDAs are moderate gain antennas with a typical average gain of 5 $\mathrm{dBi}$ [2-4]. Low gain variation over a wide range of operating frequency is also an important aspect utilized in a variety of applications $[1,5]$.

In the construction of modern systems with shrinking sizes, antenna dimension is an important factor to be considered which calls for the achievement of required performance characteristics at smaller sizes. Printed antennas are widely used for many communication systems due to having low-weight, smaller sizes, and low manufacturing cost. Therefore, for many applications, printed (planar) version of LPDAs is a more suitable option than standard LPDAs [3, 6, 7].

Maximum lateral size of printed LPDAs is proportional to the minimum frequency of operation. If the application space for an application in use is not sufficient for the employment of a traditional printed LPDA, it is necessary to come up with a smaller antenna design with satisfactory performance characteristics [6].

A review of literature on the subject matter shows a variety of size-reduced LPDAs [9-12]. In this study, the

Mehmet C. Özgönül is with the Graduate School of Natural and Applied Sciences, Izmir Institute of Technology, Gulbahce Campus, Urla, 35430, Izmir, Turkey (phone: 90-232-4842673; e-mail: mc.ozgonul@gmail.com)

Mustafa Seçmen is with the Department of Electrical and Electronics Engineering, Yasar University, Selcuk Yasar Campus, Bornova, 35100, Izmir, Turkey (phone: 90-232-5708232; e-mail: mustafa.secmen@yasar.edu.tr)

Serdar Okuyucu is with the Department of Electrical and Electronics Engineering, Yasar University, Selcuk Yasar Campus, Bornova, 35100, Izmir, Turkey (e-mail: serdar.okuyucu@gmail.com) size reduction of the miniaturized antenna is achieved in the lateral (transverse) direction. In order to reduce the lateral size of the antenna, second order iteration semicircles are added to the dipole arms at both sides of the substrate. In this way, edge-to-edge distance of the dipoles on the LPDA is decreased in achieving a $23 \%$ size reduction in lateral size whereas the total path length over the dipole arms is kept constant. On the other hand, the size of the proposed antenna in the longitudinal direction is kept constant. Even though the proposed antenna has a smaller lateral size compared to the first reference antenna, it has a considerably higher simulated gain at $2.4 \mathrm{GHz}$ than first reference LPDA, which is similar with second reference LPDA. Operating frequency range of the proposed antenna is around $0.87 \mathrm{GHz}$ to $2.55 \mathrm{GHz}$, which covers GSM, PCS, UMTS, GPS, 3G and Wi-Fi $2.4 \mathrm{GHz}$ bands. The proposed antenna with a reduced size compared to reference LPDAs (LPDA with patches added at feed points and LPDA with Single First Order SemiCircle Iteration and Feed Point Patches) also shows improved operational characteristics in terms of return loss and gain variation at a wider bandwidth. The reference antennas, with its details presented in Section II, have been previously designed and manufactured [8].

The following parts of the paper are structured in four sections. In Section II, structural specifications of reference antennas, and corresponding operational performance characteristics are presented. Section III describes the structural properties of the proposed antenna along with a comparison of measured and simulated performance with those obtained from reference antennas. Finally, conclusions are drawn in Section IV.

\section{REFERENCE ANTENNAS}

LPDA with patches added at feed points [8] is chosen as the first reference LPDA of this study. This antenna has dimensions of $152 \mathrm{~mm} \times 124 \mathrm{~mm}$ and feed line width of 3 $\mathrm{mm}$. In the initial step of the construction, a standard planar log-periodic dipole antenna is designed earlier on an FR4 dielectric substrate with the thickness of $1.6 \mathrm{~mm}$. The values of relative permittivity $\left(\varepsilon_{r}\right)$ of 4.3 and loss tangent $(\tan \delta)$ of 0.02 at $1 \mathrm{GHz}$ are performed in the simulations carried out by CST Microwave Studio 2017. Table 1 presents the design parameters of the antenna where, $\tau$ is the scaling factor, which gives periodicity relationship of the antenna dimensions, $\sigma$ is the spacing factor, $2 \alpha$ is the aperture angle, $\mathrm{N}$ is the number of dipoles, $\mathrm{L}$ and $\mathrm{W}$ are length and width of the longest dipole, respectively. In the design of the antenna, log periodic antenna formulations [1, 13] are used with some 
modifications [14]. In order to design the LPDA, $\alpha$ and $\tau$ are generally selected in the range of $10^{\circ} \leq \alpha \leq 45^{\circ}$ and $0.7 \leq \tau \leq 0.95$. If $\alpha$ increases; $\tau$ decreases, and vice versa [1]. Dipoles are located on both sides of the dielectric substrate in a symmetrical fashion. For the selected value of $\tau$ at $\tau=0.88$ [15], the spacing factor is chosen to be $\sigma=$ 0.06 , which gives an approximate directivity value of 6.8 $\mathrm{dBi}$ obtained from a graph of constant directivity versus $\sigma$ and $\tau$ for $\log$ periodic dipole array $[1,13]$.

TABle 1: Design Parameters of THe PRINTEd LPDAs

\begin{tabular}{|c|c|c|}
\hline $\boldsymbol{\tau}$ & $\boldsymbol{\sigma}$ & $\boldsymbol{\alpha}$ \\
\hline 0.88 & 0.06 & $26.6^{\circ}$ \\
\hline $\mathbf{N}$ & $\mathbf{L}$ & $\mathbf{W}$ \\
\hline 11 & $73.5 \mathrm{~mm}$ & $4 \mathrm{~mm}$ \\
\hline
\end{tabular}

In the following step, the standard LPDA is modified by adding rectangular patches as shown in Fig. 1 at the feed point of the antenna by keeping all other dimensions of the standard LPDA constant. The insertion of these patches, which may also be called as feed point patches, is a widely used method to increase the bandwidth of microstripbased antennas $[3,6,16]$. This process is also observed to increase the operational frequency bandwidth of the LPDA. The physical manufacturing of the mentioned (first reference) antenna is depicted in Fig. 2.

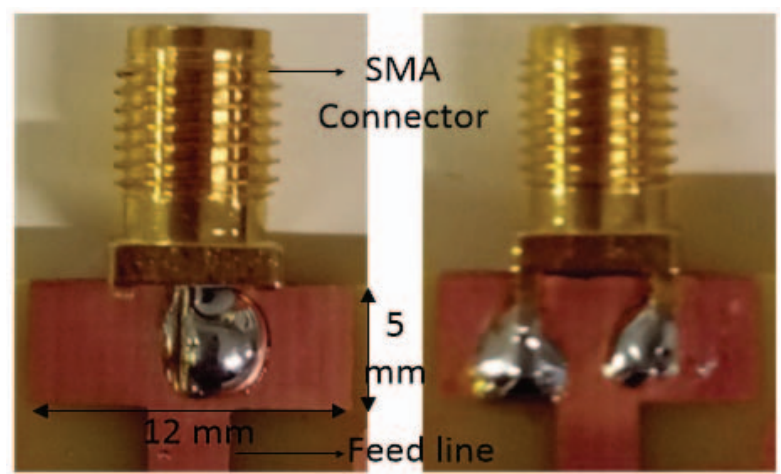

Fig. 1. Structure and dimensions of the patches at feed points of the first reference LPDA.

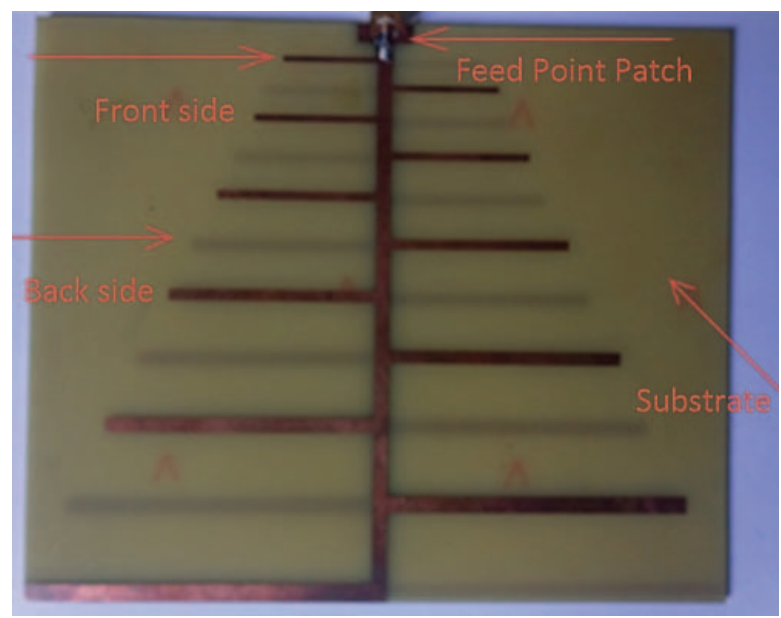

Fig. 2. The picture of the prototype of the first reference LPDA.

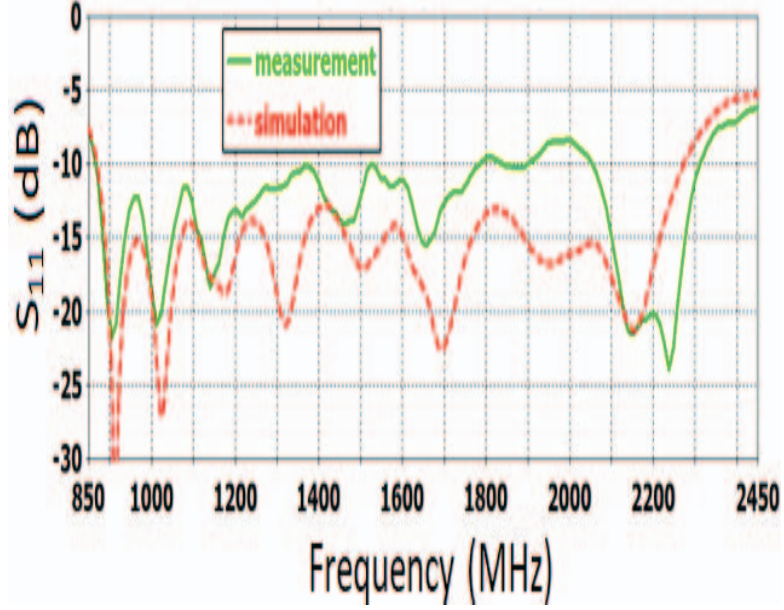

Fig. 3. The simulation and measurement results for reflection coefficients of the first reference LPDA.

The simulated and measured reflection coefficients of the antenna are given in Fig. 3. According to the results in Fig. 3, the operating frequency range of the LPDA with patches added at feed points is almost from $0.87 \mathrm{GHz}$ to $2.28 \mathrm{GHz}$.

LPDA with Single First Order Semi-Circle Iteration and Feed Point Patches [8] is the second reference antenna (as shown in Fig. 4) used in this study. In the construction of this reference antenna, first order iteration semi-circle shape dipole is employed in the printed LPDA with patches added to feed points as was done for first reference antenna. For the longest dipole with the same width of $4 \mathrm{~mm}$ as in Table 1, the radius of circle is $7.8 \mathrm{~mm}$ and the straight parts have $24.5 \mathrm{~mm}$ length. Therefore, total path length for the longest dipole becomes $2 \times 24.5$ $\mathrm{mm}+\pi \times 7.8 \mathrm{~mm} \approx 73.5 \mathrm{~mm}$, which is almost equal to the longest dipole of the first reference antenna ( $\mathrm{L}$ value in Table 1). In this way, the total length of the antenna is decreased to $133.5 \mathrm{~mm}$ in achieving a lateral size reduction at around $14 \%$ while the size of the antenna in the longitudinal direction is kept constant.

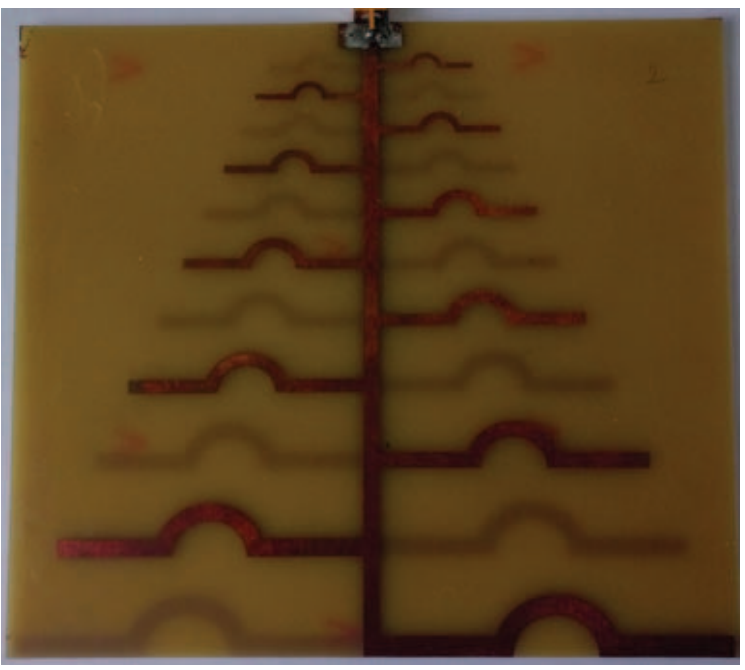

Fig. 4. The picture of the prototype of the second reference LPDA. 
The reflection coefficient results of the second reference antenna are shown in Fig. 5. It is demonstrated that working frequency range of the second reference antenna is wider than that of the first reference antenna and covers the frequency range of $0.9-2.4 \mathrm{GHz}$.

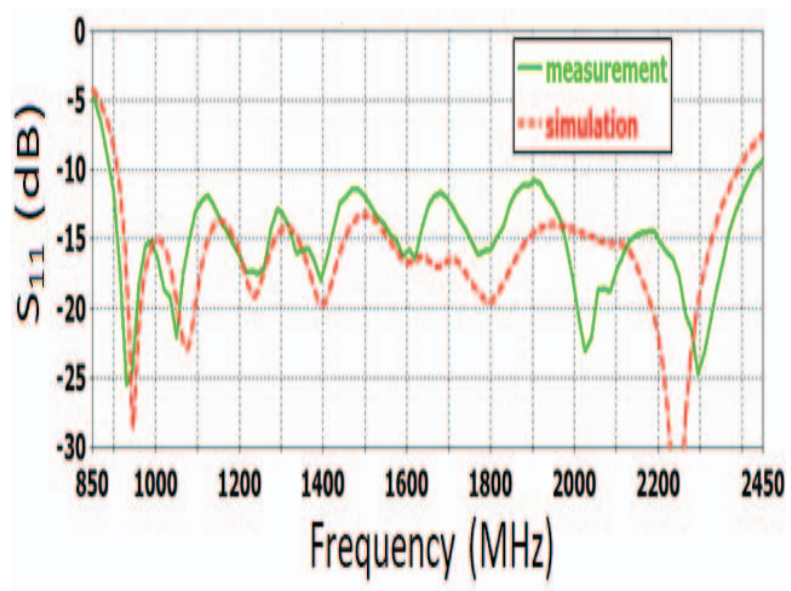

Fig. 5. The simulation and measurement results for reflection coefficients of the second reference LPDA.

III. The Design of Log Periodic Dipole Antenna WITH SECOND ORDER SEMI-CIRCLE ITERATION AND FEED POINT PATCHES

The main purpose of this study is to obtain an LPDA having smaller lateral size than the two previous reference antennas. In doing so, size reduction is achieved by decreasing the edge-to-edge distance of dipole arms. While reducing the edge-to-edge distance, the total length of conductive dipole arms (path length) is kept at the same value as in the reference antennas. No size change is made in the total longitudinal dimension of the antenna.

In the proposed antenna, size reduction is achieved by inserting second order semi-circles at each dipole arm. The structure and dimensions of the longest dipole arm of the proposed antenna are indicated in Fig. 6. Second order iteration is performed based on a study of fractal theory [1, 11] and contains additional semi-circles with smaller diameters than $15.6 \mathrm{~mm}$ (diameter of the first order semicircle) inserted on straight and curved parts. These semicircles have diameters of $5.2 \mathrm{~mm}$ on the straight part and $3.12 \mathrm{~mm}$ on the curved part for the longest dipole arm of the second reference design as shown in Fig. 6. In this way, a total of $29 \mathrm{~mm}$ shortening in the longest dipole is achieved when compared with first reference antenna.

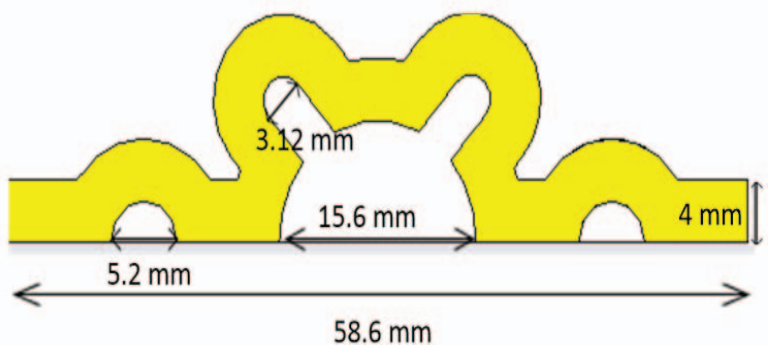

Fig. 6. The second-order size-reduced structure for the arm of the longest dipole on the front side of substrate.
The size reduction in the lateral direction is acquired by employing the same approach used for the model shown in Fig. 6 where dipole dimensions are scaled down along with the radii of the added circles. The physically constructed model of the size reduced LPDA antenna is shown in Fig 7. The proposed antenna is found to have lateral dimension of about $123.5 \mathrm{~mm}$, where corresponds to a reduction of $23 \%$ and $8 \%$ in lateral dimension with respect to the first and second reference antennas.

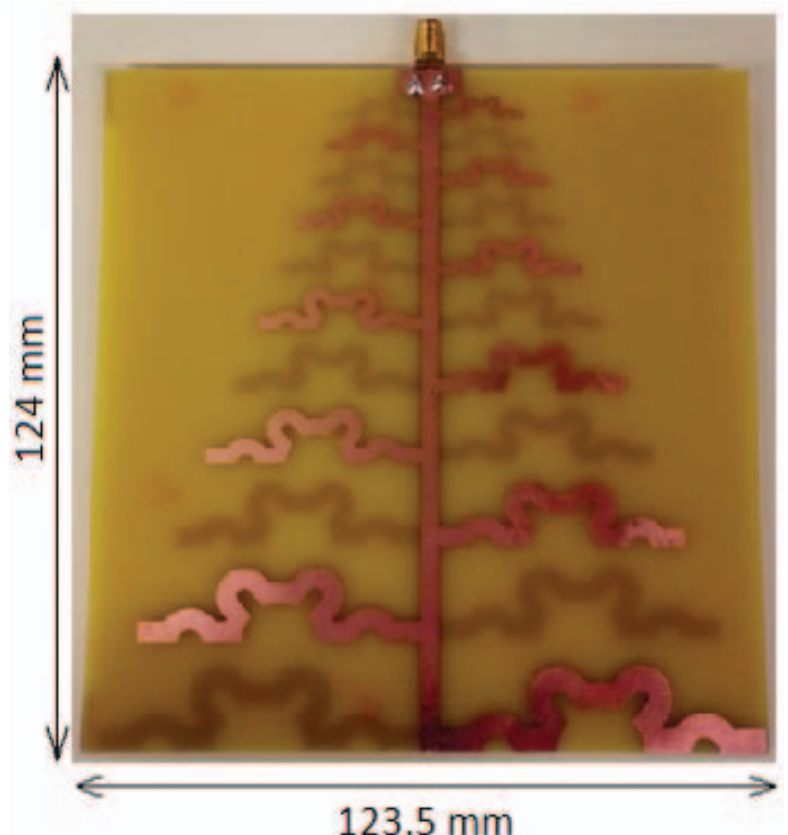

Fig. 7. The picture of the prototype of the proposed LPDA.

Following a laboratory measurement of return loss for the proposed antenna, a comparison is made between simulation and measurement results. The measurement results reveal that the $\left|S_{11}\right|$ of the antenna is almost below $-10 \mathrm{~dB}$ in the frequency range from $0.87 \mathrm{GHz}$ to $2.55 \mathrm{GHz}$ as presented in Fig. 8 .

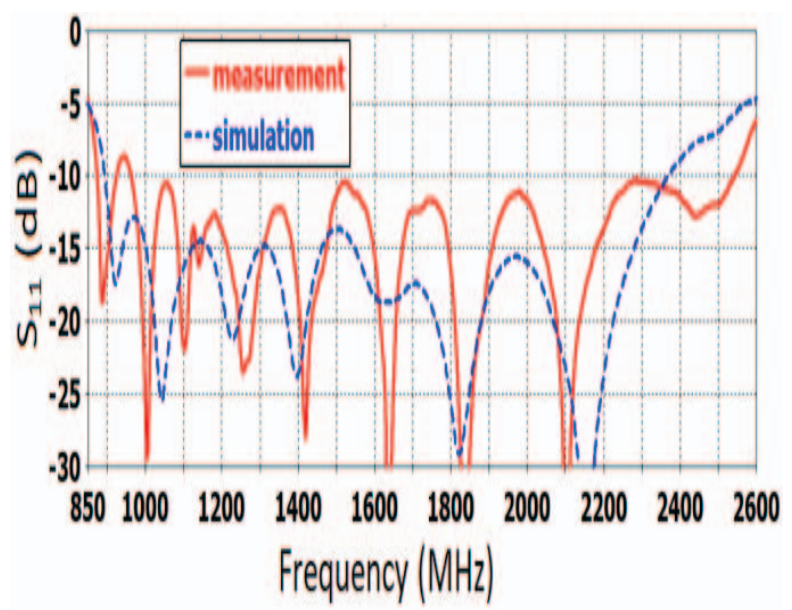

Fig. 8. The simulation and measurement results for reflection coefficients of the proposed LPDA. 
The comparison of measured $\left|\mathrm{S}_{11}\right|$ of the proposed and reference antennas is also given in Fig. 9, where the proposed LPDA is observed to have the widest frequency band among three antennas in consideration with the frequency band of 0.87-2.55 GHz.

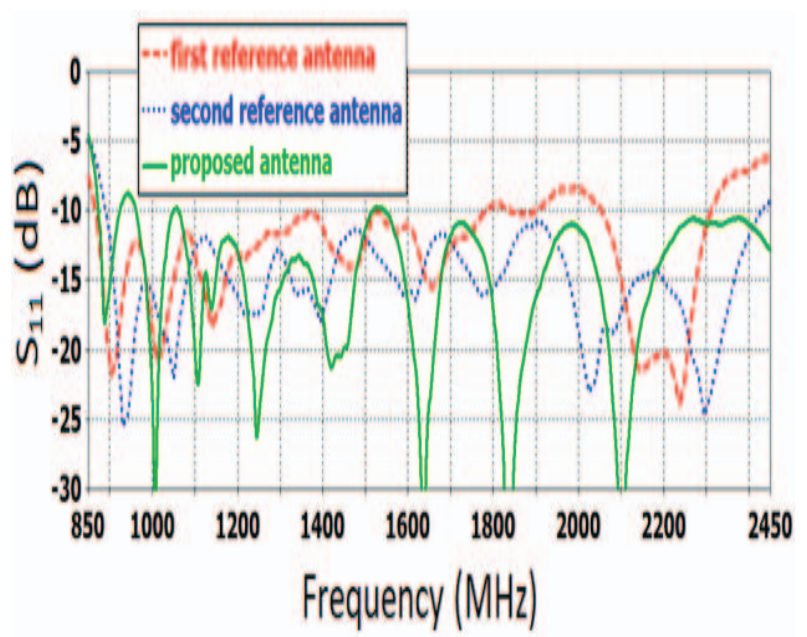

Fig. 9. The measurement results for reflection coefficients of the first, second reference and proposed antennas.

The gain values of all LPDAs are also examined such that the values obtained from simulation results at certain frequencies of interest are given in Table 2. At lower frequencies of operation, the proposed antenna has less gain than the reference LPDAs; however, it can be still considered as sufficient for the applications. The gain values at the upper edge of the frequency bandwidth (especially at $2.4 \mathrm{GHz}$ ) are higher than the first reference antenna and similar with the second LPDA except for 2.25 $\mathrm{GHz}$. For the proposed antenna, gain variation is found to be less than $2.8 \mathrm{dBi}$ within the respective operating frequency range, which is the best as compared to the first and second reference antennas with more than $4.1 \mathrm{dBi}$ and $2.9 \mathrm{dBi}$ gain variation values, respectively. Moreover, the widest range of operational frequency is obtained by the proposed antenna at lowest lateral dimension when compared with the reference antennas.

Table 2: The Simulated Gain Values at Certain FREQUENCIES FOR THREE LPDAS

\begin{tabular}{|c|c|c|c|}
\hline $\begin{array}{c}\text { Frequency } \\
(\mathrm{GHz})\end{array}$ & $\begin{array}{c}\text { First } \\
\text { reference } \\
\text { LPDA }\end{array}$ & $\begin{array}{c}\text { Second } \\
\text { reference } \\
\text { LPDA }\end{array}$ & $\begin{array}{c}\text { Proposed } \\
\text { LPDA }\end{array}$ \\
\hline 0.9 & $5.57 \mathrm{dBi}$ & $3.93 \mathrm{dBi}$ & $3.46 \mathrm{dBi}$ \\
\hline 1 & $5.42 \mathrm{dBi}$ & $5.28 \mathrm{dBi}$ & $4.72 \mathrm{dBi}$ \\
\hline 1.5 & $5.41 \mathrm{dBi}$ & $5.55 \mathrm{dBi}$ & $5.44 \mathrm{dBi}$ \\
\hline 2 & $5.04 \mathrm{dBi}$ & $5.41 \mathrm{dBi}$ & $5.40 \mathrm{dBi}$ \\
\hline 2.25 & $3.29 \mathrm{dBi}$ & $4.33 \mathrm{dBi}$ & $3.91 \mathrm{dBi}$ \\
\hline 2.4 & $1.45 \mathrm{dBi}$ & $2.64 \mathrm{dBi}$ & $2.65 \mathrm{dBi}$ \\
\hline
\end{tabular}

\section{CONCLUSION}

A novel printed log periodic dipole antenna (LPDA) with second order semi-circle iteration and feed point patches has been presented. The presented antenna is shown to have a wide range of operating frequency covering GSM, PCS, UMTS, GPS, $3 \mathrm{G}$ and Wi-Fi $2.4 \mathrm{GHz}$ bands. By the employment of second order iteration, size reductions are achieved by a factor of $23 \%$ and $8 \%$ over that of the first and second reference antennas, respectively. The proposed antenna is shown to cover the widest frequency range among the two reference LPDAs with the best gain variation at the respective frequency band of operation.

\section{REFERENCES}

[1] C. A. Balanis, "Antenna Theory: Analysis and Design", 4th ed., New York: John Wiley \& Sons, 2016, pp. 619-648.

[2] G. A. Casula, P. Maxia, G. Mazzarella, and G. Montisci, "Design of a printed log-Periodic dipole array for ultra-wideband applications", Progress in Electromagnetics Research C, vol. 38, pp. 15-26, Jan. 2013.

[3] G. Bozdag and A. Kustepeli, "Subsectional tapered fed printed LPDA antenna with a feeding point patch", IEEE Antennas and Wireless Propagation Letters, vol. 15, pp. 437-440, June 2015.

[4] M. Secmen, "Scattering method for resonance region target recognition: Application to small-scale airplane targets with measured data", in European Conference on Antennas and Propagation (EUCAP), Lisbon, Portugal, 2015, pp. 1-5.

[5] W. L. Stutzman and G. A. Thiele, "Antenna Theory and Design", 3rd ed., New York: John Wiley \& Sons, 2013, pp. 251-260.

[6] G. Bozdağ, "Novel microstrip antennas for multiband and wideband applications", M.S. thesis, Electron. and Communi. Eng., Izmir Institute of Tech., Izmir, Turkey, 2014.

[7] G. A. Casula, P. Maxia, and G. Mazzarella, "A printed LPDA with UWB capability", in International Workshop on Antenna Technology (iWAT), Lisbon, Portugal, 2010, pp. 1-4.

[8] M. C. Özgönül, and M. Seçmen, "Size-reduced printed log periodic dipole antenna with single first order semi-circle iteration and feed point patches", accepted for International Conference on Electrical and Electronics Engineering (ELECO), Bursa, Turkey, Nov. 2017.

[9] R. X . Liang, P. F. Zhang, S. X. Gong, and F.W. Wang, , "A novel method for RCS reduction of the printed log periodic dipole array", Journal of Electromagnetic Waves and Applications, vol. 26, pp. 1631-1640, Aug. 2012.

[10] A. Kajla and R. Kushwaha, "Design a log periodic fractal koch antenna for multiband applications", International Journal of Advance Research In Science And Engineering, vol. 2, pp. 76-81 Oct. 2013.

[11] D. F. Chairunnisa, D.F. Sihaloho, and A. Munir, "Size reduction of printed log-periodic dipole array antenna using fractal koch geometry", International Journal on Electrical Engineering and Informatics, vol. 7, pp. 226-236, June 2015.

[12] L. Song, Y. Nie, and J. Wang, "A novel meander line microstrip log-periodic dipole antenna for dual-polarized radar systems", Progress In Electromagnetics Research Letters, vol. 56, pp. 123128, Jan., 2015.

[13] R. Carrel, "The design of log-periodic dipole antennas" 1958 IRE International Conventional Record, vol. 9, pp. 61-75, March 1961.

[14] D. M. Pozar, "Microwave Engineering", 4th ed., United States: John Wiley \& Sons, 2005, pp. 141-150 .

[15] E. Ávila-Navarro, J. M. Blanes, J. A. Carrasco, J. Reig, and E. A. Navarro, "A New Bi-Faced Log Periodic Printed Antenna," Microwave and Optical Technology Letters, vol. 48, pp. 402-405, Dec. 2005.

[16] G. Bozdag and M. Secmen, "Eğimli Beslemeli Geniş Bantlı Basılı Papyon Anten Tasarımı", in URSI-TÜRKIYE'2016 VIII. Bilimsel Kongresi, Ankara, Turkey, 2016, pp. 1-3. 